\title{
LETTRE DE M. L'ABBÉ POTTIER
}

en réponse aux observations présentées sur le premier Congrès de la ligue démocratique belge.

$\mathrm{D}^{\mathrm{s}}$

ANS la dernière livraison de la Revue bénédictine nous avons émis, d'après le compte-rendu des journaux, quelques réserves sur les travaux du premier congrès de la Ligue démocratique belge.

M. l'abbé Pottier, mis en cause dans deux de ces observations, nous fait l'honneur de nous adresser à propos de cet article la lettre suivante que nous nous empressons d'insérer dans nos colonnes.

Au T. R. Dom Laurent Janssens.

Liége, le 29 novembre $189 \%$

Mon très Révérend Père,

D'un article que vous avez publié dans votre excellente Rezue bénédictine de Maredsous, il pourrait peut-être résulter dans l'esprit de vos lecteurs qu'au Congrès de la Ligue démocratique de Bruxelles, je me suis prononcé en faveur du droit strict de l'ouvrier d'entrer en participation des bénéfices réalisés par son patron sous le régime du contrat de salaire.

Comme on a fait parfois autour de mon nom beaucoup plus de bruit que je ne l'aurais souhaité, je crois ne point devoir laisser subsisster d'équivoque sur ce que j'ai défendu.

Après la lecture du rapport sur la question dont je parle, j'ai signalé une incöhérence de rédaction entre la conclusion et les motifs. Interpellé par Monsieur Levie, qui présidait, sur le point disavoir si mon observation ne portait que sur la forme, j'ai répondu affirmativement et j'ai ajouté que je n'entendais point par là émettre un jugement sur le fond. C'est à cela que s'est bornée toute mor intervention à la séance où l'on a lu le rapport. A la séance sui vante, j'ai fait remarquer que le salaire juste pouvait dépasser le salaire minimum; que quand il le dépassait, le surplus constituait une espèce de participation due en vertu de la stricte justice. J'ai mème développé la nature du contrat de salaire pour appuyer mon assertion et exclure toute autre participation aux bénéfices comme 
exigée par la stricte justice. En exiger une autre, c'est confondre le contrát de salaire avec le contrat d'association.

C'est là la doctrine que j'ai rappelée brièvement dans la discus. sion d'une question mise par le bureau du Congrès à l'ordre du jour, doctrine qu'a: surplus j'expose ex professo et in extenso dans le traité du contrat de louage d'œuvres.

Il est une autre question sur laquelle il y a aussi équivoque : c'est celle des syndicats.

Que ni mes amis ni moi ne veuillons défendre ni créer des syndicats de guerre, c'est ce que je crois inutile de rappeler. Il est aussi inutile d'affirmer que nous ne cherchons qu'arriver à la paix entre le capital et le travail.

Tout autant que nos amis qui veulant d'un seul coup mettre ensemble, sans aucune organisation préalable, patrons et ouvriers pour régler les droits aussi bien que les devoirs des deux parties, je crois nécessaires les syndicats mixtes comme but d attendre.

Par quel moyen atteindra-t-on ce but? Voilà la question Avec les cardinaux Manning et Gibbons,avec Monsieur Decurtius, a vec Monsieur le comte de Mun qui, malgré ce qu'il ait pu penser précédem. ment, $m$ 'a fait cette année même les déclarations les plus explicites sur ce sujet, avec nos amis les chefs des 5000 antisocialistes de Gand, avec ceux de Lokeren, avec ceux de Charleroi, je crois que pour arriver à régler pratiquement et effectivement les intérêts tcls qu'ils se présentent aujourd'hui, des deux parties en cause, il faut, surtout dans la grande industrie, permettre aux ouvriers de repren. dre leur indépendance en les groupant en syndicats. Ainsi unis, ils pourront, par leurs délégués se rencontrant dans une institution permanente avec les délégués des patrons, constituer le syndicat mixte auquel appartiendra d'examiner et de résoudre toutes les questions qui regardent à la fois le capital et le travail.

Je crois même, qu'étant donnée l'étendue du marché et l'organisation du travail sous le régime de la Société Anonyme, la corporation dans la grande industrie actuelle n'est possible que de cette façon. C'est ainsi que semble avoir jugé notre nouvelle loi sur les conseils de l'industrie et du travail ; il reste maintenant à étendre l'esprit et les attributions de cette loi ; il rește surtout à faire pénétrer dans les mœurs ce qui n'est encore que dans le principe de la loi. C'est le grand objectif qui s'impose à l'apostolat catholique, afin que les nouvelles organisations professionnelles soient chrétiennes, dans leurs principes,dans leur fonctionnement et dans leur but.

On dit que nous préparons des recrues pour les rangs de l'armée 
socialiste. Je suis convaincu que ces appréhensions, qui se tournent parfois en pénibles accusations, ne sont point fondées. Nulle part jusqu'ici on n'a fait l'expérience de syndicats chrétiens de ce genre. Ou plutôt, je me trompe; les quelques expériences faites jusqu'ici donnent tort au pessimisme de quelques-uns. Les antisocialistes de Gand, les coopérateurs de Charleroi, les syndiqués de la région de Liége n'ont été jusque maintenant que de solides remparts contre la révolution.

Plusieurs fois, hélas! j'ai été victime de reportages inexacts qui peuvent avoir fait du mal à ma réputation et à ma petite action sociale. Comme j'ai le devoir de défendre l'une et l'autre, je vous. prierai de vouloir bien publier ces quelques lignes dans votre prochain numéro de la Revue. J'espère que les journaux qui à la suite de votre article ont cru devoir s'occuper de moi, voudront bien à leur tour insérer ces observations.

Daignez agréer, mon très Révérend Père, avec mes remerciments anticipés, l'hommage de mon plus profond respect.

A. Pottier, professeur au Grand Séminaire de Liége.

Liége le 29 nov. 1892 .

Je répondrai en peu de mots à la lettre de M. l'abbé Pottier.

Si mes observations relatives aux travaux du Congrès de la Ligue democratique n'avaient eu d'autre effet que de provoquer cet échange de vues, je me féliciterais de les avoir émises. Il m'en coutait beaucoup, je l'avoue, de combattre ouvertement dans une question aussi importante que la justice du salaire, le sentiment d'un homme dont je respecte si haut le caractère,le talent et les intentions.

Si j'ai pu mal interpréter la thèse de M.l'abbé Pottier, faute de posséder les documents authentiques, je vois avec bonheur que le docte professeur se range à mon sentiment. Il affirme nettement qu'on ne peut confondre le contrat de salaire avec le contrat d'association. Or, le contrat de salaire, tel qu'on le trouve dans la parabole des vignerons, a toujours été regardé par tous les théologiens comme juste, moyennant une base équitable de rémunération d'après les principes de l'égalité d'échange; donc le contrat d'association n'est pas requis par la justice.

Sans doute, on pourrait, suivant les circonstances, organiser certains travaux d'après le principe d'association plutôt que de salaire; à condition d'avoir une population ouvrière stable et groupée en corps; mais alors la participation aux pertes s'imposerait au même titre que la participation aux bénéfices. Vouloir, en cas de bénéfices, 
s'en tenir au contrat d'association, et, en cas de pertes, se rejeter sur celui du salaire et même d'un salaire augmenté, comme un journaliste n'a pas craint de le soutenir récemment, c'est vraiment jouer double jeu et offenser une des règles fondamentales de la logique.

Le grand principe demeure celui-ci : le travail de l'ouvrier est une valeur qui demande une rémunération exacte. Comment l'estimer le plus sûrement? En sus du minimum fixé par le droit naturel, minimum que Léon XIII appelle l'élément nécessaire, et qu'on pourrait aussi appeler subjectif, il y a la valeur industrielle du travail, l'élément objectif. Plus l'ouvrier est habile, plus la situation générale de l'industrie est florissante, plus aussi cet élément objectif croîtra, dans le premier cas à cause de la valeur plus grande du travail spécial fourni par l'ouvrier, dans le second cas à cause de la plus grande value du travail en général. Cette plus grande value, on peut, je l'avoue, dans une certaine mesure, la considérer comme « une espèce de participation due en vertu de la stricte justice $\Downarrow$, comme s'exprime M. l'abbé Pottier. Cependant, pour ne pas donner prise à de fausses déductions, il est beaucoup plus logique de ne voir dans cette plus value qu'une rémunération équivalente du travail. Comme tous les éléments qui concourent au fonctionnement industriel, le travail a ses hauts et ses bas, suivant que l'industrie est prospère ou en souffrance, pourvu toutefois que ces bas ne descendent jamais en dessous du minimum exigé par l'élément nécessaire ou subjectif, basé sur la loi naturelle. Tous les efforts des économistes chrétiens doivent tendre à garantir au salaire ce minimum stable. Mais de là à proclamer la participation aux bénéfices comme requise ex justitia, il y a un abìme.

Et qu'on n'objecte pas que sans l'ouvrier le capital ne peut pas produire, que par conséquent le travail est l'associé du capital. Sans typographes un écrivain ne peut publier aucun ouvrage; faudra-t-il les rémunérer suivant les honoraires des auteurs pour lesquels ils travaillent? Sans souffleur un organiste, si artiste soit-il, ne peut donner aucun son; M. Widor devra-t-il associer son souffleur aux bénéfices de ses concerts? Ce sont là des objections spécieuses peut-être, mais qui ne tiennent pas devant une analyse raisonnée.

Et puis, comment étendre cette notion d'associé à tous les genres de travaux et de services? Un employé de ferme dépendra-t-il pour son,salaire du rendement de la moisson? une fille de magasin, des bénéfices de la firme? un clerc de notaire, de ceux de son patron? un garde-train, des recettes du département des chemins de fer? 
De plus la prospérité d'une maison est souvent le fruit de longs surnrts stériles. Voilà donc une foule d'ouvriers qui ont travaillé au minimum dans les années de peine, et d'autres, qui, tout en se donnant beaucoup moins de mal, récoltent les fruits de ce que leurs devanciers ont semé. Sic vos non vobis!

Que dire encore de la part prépondérante qui revient à la direction d'une affaire dans les bénéfices qu'elle réalise? Le rendement de toute une année peut dépendre d'une seule combinaison, d'un heureux calcul, d'une invention nouvelle, de mille choses, en un mot, dont le mérite revient uniquement à la direction, que ce soit le patron lui-même ou que ce soit le conseil d'administration. Dès lors, n'est-ce pas manifestement dépasser les exigences de la justice que d'étendre de droit aux ouvriers les fruits d'efforts auxquels ils sont étrangers à moins que, groupés en corps, ils, n'entrent dans la direction? Tout autre est la plus value du travail provenant de la prospérité générale de l'industrie. Celle-là, on l'a vu, les touche de droit parce qu'elle modifie, si je l'ose dire, le capital même de l'ouvrier.

En effet, tout ouvrier connaissant un métier, tout homme capable de remplir n'importe quelle charge, représente un capital correspondant à la valeur de ce travail, de cette charge. Quand l'industrie prospère, ce capital est en hausse, à peu près comme la valeur des terres s'accroît avec la prospérité de l'agriculture. Trouver dans ce point de vue quelque chose de brutal qui froisse la dignité de l'hoiame, c'est faire du sentiment hors de propos. Saint Thomas et les meilleurs théologiens ne parlaient pas autrement. Qui osera leur reprocher d'avoir méconnu la dignité du travailleur? Pourvu, je le répète, que l'élément nécessaire soit scrupuleusement maintenu, l'ouvrier n'a rien à craindre de cette manière d'estimer son travail ; au contraire, il y gagnera ; sa rémunération sera plus stable et récompensera plus sûrement son mérite.

Ces considérations suffiront comme complément à celles que j'ai exposées déjà sur cette question, notamment dans mon dernier article. J'avais eu l'intention de les développer davantage; mais, en présence des déclarations de M. l'abbé Pottier, je crois inutile de m'y arrêter plus longuement. A ceux qui insisteraient sur le caractère de justice stricte de la participation aux bénéfices, je pourrai me contenter d'opposer le sentiment du docte professeur.

- Je ne dirai qu'un mot de la seconde question traitée dans la lettre de mon révérend correspondant. Je souhaite que tous les chefs de la ligue démocratique travaillent autant à la pacification des esprits qu'au redressement des griefs populaires. Loin de moi de suspecter 
les intentions. Ma devise sera toujours celle de Dupanloup : $\$ \mathrm{Je}$ cherche ce qui rapproche et non ce qui sépare. "Loin de moi surtout de vouloir démolir l'apostolat de personne. Cependant, plus les intentions sont à mes yeux excellentes, plus je redoute les moindres manques de prudence qui risquent de les faire suspecter ; plus l'apostolat ouvrier est nécessaire, plus je souhaite qu'aucune ardeur excessive ne vienne le compromettre. Je ne juge pas, j'exprime un vœu.

Quelque légitimes que soient les griefs du peuple, quelque urgente que soit l'organisation chrétienne du travail, je ne puis voir sans crainte les droits des ouvriers caressés au détriment de leurs devoirs, ni les devoirs des patrons mis en relief au détriment de leurs droits.

Droit et devoir, liberté et autorité, ces choses ne doivent jamais être séparées. « Car l'autorité, dit Lacordaire, est aussi une liberté, et quiconque voulant défendre celle-ci, attaque celle-là, ne sait ni ce qu'il dit, ni ce qu'il fait. L'autorité est une partie intégrante de la liberté, comme le devoir rentre dans le droit par une corrélation manifeste, puisque le droit d'un homme entraîne nécessairement le devoir d'un autre. C'est pourquoi les chartes civiles, aussi bien que la grande charte évangélique, consacrent en même temps le droit et le devoir, la liberté et l'autorité. Toute main qui les sépare les anéantit, et jamais un peuple qui ne les vénère pas au même titre ne sera capable de devenir un peuple libre (I). 》

Il faut des ménagements inouis pour traiter en public des ques. tions aussi délicates que les droits de la classe ouvrière ( $\left.{ }^{2}\right)$. Pour pacifiques que soient les intentions, on ne doit jamais s'y aventurer devant un auditoire quelconque avant de connaître à fond les conditions locales tant des patrons que des ouvriers. Sinon la parole la plus éloquente, la plus docte, la plus apostolique, risque de provoquer des heurts fâcheux et de déplorables équivoques.

Moins ces problèmes brûlants seront livrés en pâture aux masses, moins ces questions de devoir et de justice seront tranchées dans la presse populaire par des écrivains improvisés qui manquent de la science nécessaire pour les aborder, mieux j'augurerai des efforts, au reste très louables, de notre mouvement social catholique.

Cela dit, je ne puis qu'applaudir aux déclarations de M. l'abbé Pottier, et mon plus ardent désir est de voir les catholiques s'unir de plus en plus sous l'étendard de l'Encyclique Rerum novarum interprêtée sans exagération, sans doute, mais aussi sans atténuation.

D. LaURent JansSEns.

x. Oraison sunèbre d'O'Connell. - 2. Donoso Cortès insistait sur cette vérité. 